\title{
Interactive Flow Behaviour and Heat Transfer Enhancement in a Microchannel With Cross-flow Synthetic Jet
}

\author{
D Jagannatha, T. T Chandratilleke*, R Narayanaswamy \\ Department of Mechanical Engineering \\ Curtin University of Technology \\ GPO Box U1987, Perth WA 6845, Australia \\ Email: t.chandratilleke@curtin.edu.au
}

\begin{abstract}
This paper examines the effectiveness in combining a pulsating fluid jet for thermal enhancement in microchannel heat sinks. The proposed arrangement utilises an oscillating diaphragm to induce a high-frequency periodic fluid jet with zero net mass output at the jet orifice hence, termed "synthetic jet". The pulsed jet interacts with the fluid flow through microchannel passages altering their flow characteristics. The present study develops a 2-dimensional finite volume numerical simulation based on unsteady Reynolds-averaged Navier-Stokes equations for examining the microchannel-synthetic jet flow interaction. For a range of parametric conditions, the behaviour of this periodic flow with its special features is identified and the associated convective heat transfer rates are predicted. The results indicate that the pulsating jet leads to outstanding thermal performance in microchannel flow increasing its heat dissipation rate by about 4.3 times compared to a microchannel without jet interaction within the tested parametric range. The degree of thermal enhancement is seen to grow continuously to reach a steady value in the absence of fluid compressibility. The proposed strategy has an intrinsic ability for outstanding thermal characteristics without causing pressure drop increases in microchannel fluid passages, which is identified as a unique feature of the technique. The study also examines and presents the effects of fluid compressibility on the heat removal capacity of this arrangement. The technique is envisaged to have application potential in miniature electronic devices where localised cooling is desired over a base heat dissipation load.
\end{abstract}

Keywords: synthetic jet, microchannel, electronic cooling, pulsating jet heat transfer

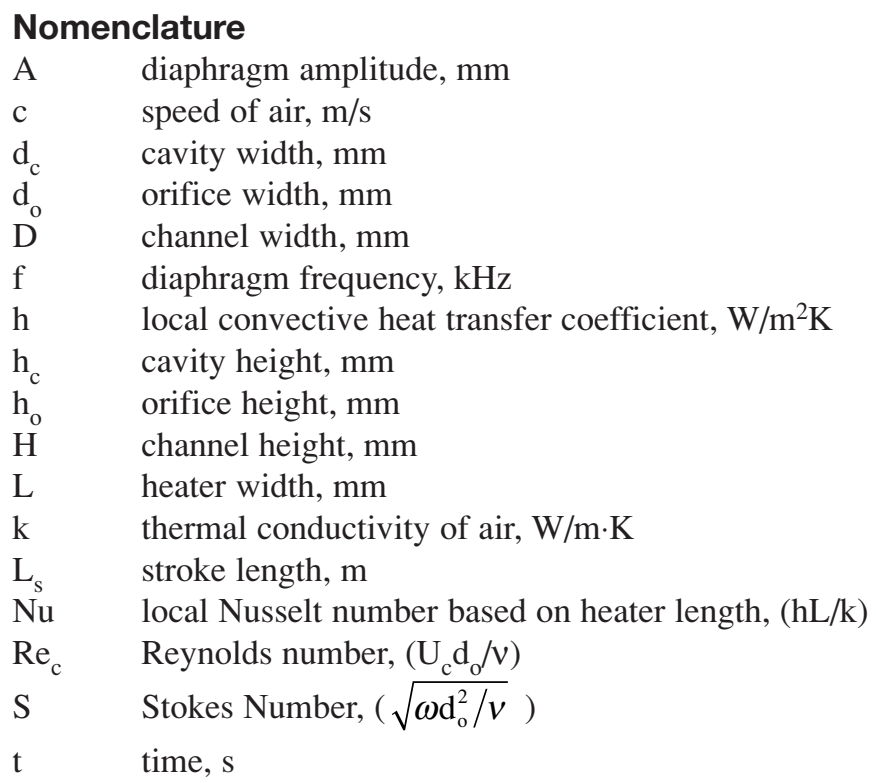


$\mathrm{T}$ time period, (1/f), $\mathrm{s}$

$\mathrm{T}_{\mathrm{b}} \quad$ bulk temperature, $\mathrm{K}$

$\mathrm{T}_{\mathrm{w}} \quad$ wall temperature, $\mathrm{K}$

$\mathrm{U}_{\mathrm{c}}^{\mathrm{w}} \quad$ characteristic velocity, $\mathrm{m} / \mathrm{s}$

$\mathrm{u}_{\mathrm{o}} \quad$ velocity through orifice, $\mathrm{m} / \mathrm{s}$

$\mathrm{V}_{\mathrm{i}} \quad$ microchannel inlet velocity, $\mathrm{m} / \mathrm{s}$

\section{Greek Symbols}

$\rho \quad$ density of air, $\mathrm{kg} / \mathrm{m}^{3}$

$\mu \quad$ dynamic viscosity of air, $\mathrm{kg} / \mathrm{ms}$

$\omega$ angular velocity, $\mathrm{rad} / \mathrm{s}$

$v \quad$ kinematic viscosity of air, $\mathrm{m}^{2} / \mathrm{s}$

\section{INTRODUCTION}

Modern lifestyle is heavily dependant on the use of microelectronic devices such as mobile phones, laptop notebooks, televisions and navigation systems. During operation, the electrical circuits characteristically generate heat within internal device components. In the absence of appropriate thermal management strategies, this heat build-up causes circuit temperatures to rise above safe operating limits of delicate semiconductor material leading to unreliable operation and thermal breakdown to reduce device-working life. Effective regulation of internal component temperatures is therefore a critical design consideration for modern electronic systems in improving application potential and performance.

A recent update by International Technology Roadmap for Semiconductors (ITRS) in 2008 has predicted a dramatic increase in the number of components integrated into a single unit, as illustrated in Figure 1. Exponential growth in applications over the past decade has motivated and intensified research to discover novel techniques to further enhance heat transfer capabilities towards meeting future demands.

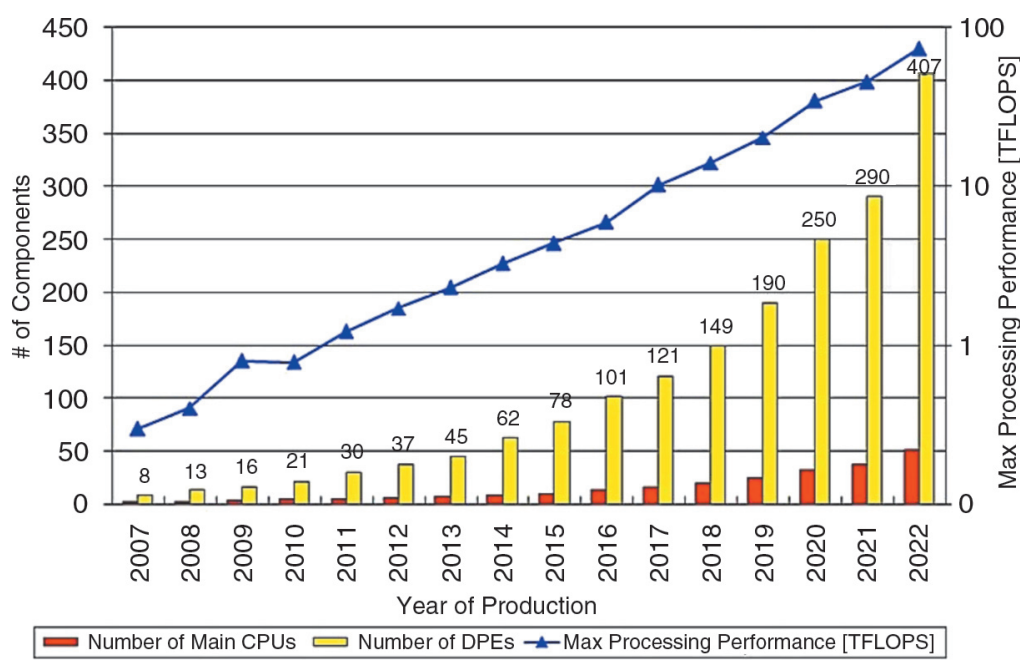

Figure 1. Component density and maximum processing performance trends -source ITRS 2008.

Microchannel behaviour is relatively well understood through established numerical modelling methods and experimentation $[1,2,3]$. The primary focus of microchannel research is to predict and validate thermal performance. Much less attention has been directed for developing effective thermal enhancement strategies for micro-scale channels. Reviewing published literature on single-phase micro and minichannel heat transfer, Steinke and Kandlikar [4] have cited that the use of internal fins in microchannels is a very promising passive enhancement option although the increased pressure drop would be a design concern.

While passive enhancement techniques have conceivable application potential, active methods may prove to be more thermally effective and relevant for future cooling needs, especially if the thermal enhancement is possible without incurring a penalty through increased pressure drop. As discussed in the sections below, the microchannel flow interrupted by a pulsating fluid jet is envisaged to be an option of this nature. 
The present study examines the performance of a new active enhancement method that can be integrated into microchannel heat sinks. As schematically shown in Figure 2, the proposed method adopts a pulsating jet mechanism called a "synthetic jet" that interacts with the flow through the microchannel whereby outstanding thermal characteristics are achieved without incurring adverse pressure drop penalties.

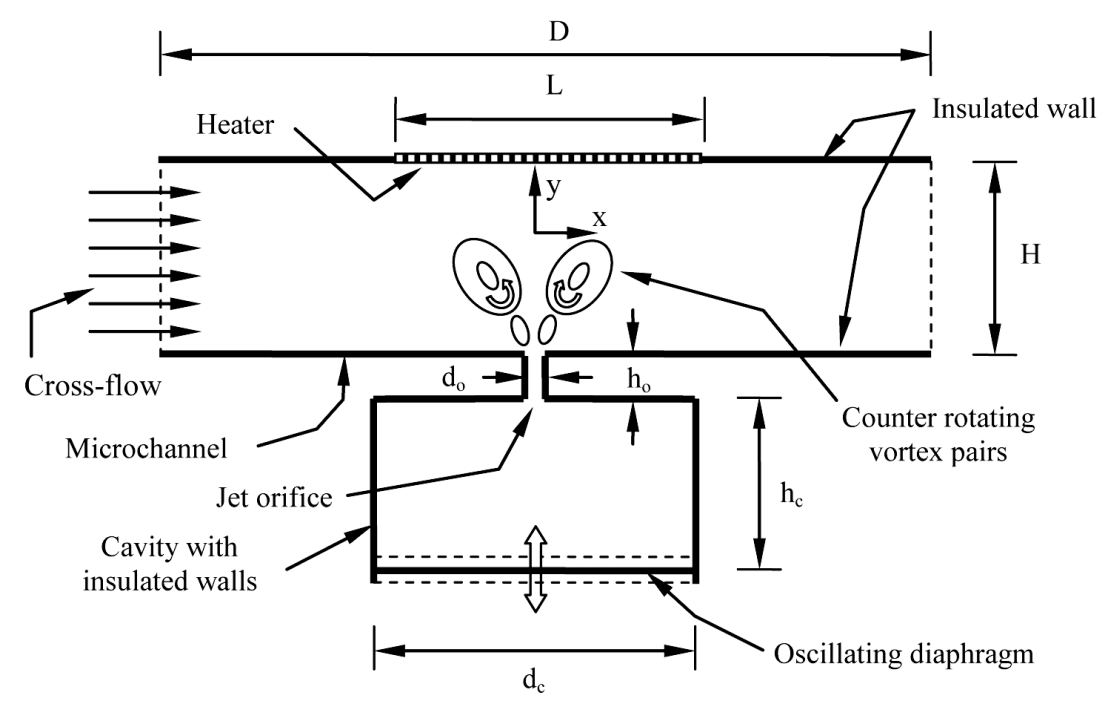

Figure 2. Schematic diagram of synthetic jet mounted on microchannel in cross-flow configuration.

The synthetic jet mechanism mounted on the microchannel comprises an oscillating diaphragm that moves back and forth at frequency (f) forcing fluid flow through a small orifice. In its inward motion, the diaphragm imparts a high-speed jet into the microchannel creating a pair of counter-rotating vortices in the surrounding fluid. When retreating, it draws fluid back into the cavity. The fluid ejected through the orifice and the vortices periodically interrupt and interact in cross flow manner with the microchannel fluid flow. Over one diaphragm cycle, the jet discharges an intense net outflow of fluid momentum into the microchannel while the net mass delivered through the orifice is zero. Therefore, this jet mechanism is known as a "synthetic jet" or Zero-Net-Mass-Flux jet.

Synthetic jets have been primarily studied in the context of pulsating jet actuators impinging on submerged surfaces in quiescent fluid media without any cross flow interactions. Such studies indicate outstanding thermal characteristics for localised cooling with synthetic jets. Significant examples of those are by Campbell et al. [5] who have demonstrated that synthetic air micro jets were effective cooling arrangements for laptop processors while Mahalingam et al. [6][7] illustrated the effectiveness of synthetic jets for high power electronic cooling by developing an integrated active heat sink based on this mechanism. Gillespie et al. [8] provide the results of an experimental investigation of a rectangular synthetic jet impinging on a unconfined heated plate exposed to the ambient where characteristics of the jet and plots of Nusselt numbers are available. Pavlova and Amitay [9] have conducted experimental studies on impinging synthetic jets for constant heat flux surface cooling and compared its performance with a jet having no velocity fluctuations known as steady or continuous jet. They concluded that for the same Reynolds number, synthetic jets provide three times more effective cooling than the corresponding continuous jets.

Aiming at flow control applications, the aerospace research studies have examined synthetic jets interacting with fluid cross flow [10,11]. However, the impact of these interactions on thermal flow characteristics has rarely been investigated for heat transfer applications where very little such knowledge currently exists. Among few reported studies addressing this shortfall, Jabbal and Zhong [12] experimentally examined a case of synthetic jet discharged from an orifice on a heated surface into a low-Reynolds number laminar flow. For this non-surface impinging jet flow, they mapped out the thermal footprint of the jet flowing over the heated surface using liquid crystal thermography. However, the investigation did not assess the impact of this synthetic jet on thermal performance. 
Utturkar et al. [13] experimentally studied synthetic jet acting parallel to the flow within a duct. The synthetic jet was placed at the surface of a heated duct wall and aligned with the bulk flow such that the jet assisted the bulk flow. In a $100 \mathrm{~mm}$ square channel with a $30 \mathrm{~mm}$ synthetic jet, they obtained a 5.5 times enhancement for a bulk flow velocity $1 \mathrm{~m} / \mathrm{s}$. This enhancement reduced to approximately 3 times when the bulk velocity was increased to of $2.0 \mathrm{~m} / \mathrm{s}$. Their restricted numerical simulation matched reasonably well with only one test condition.

Go and Mongia [14] experimentally studied the effect of introducing a synthetic jet into a low speed duct flow to emulate the confined flow within in a typical notebook. The interaction of these two flows was studied using particle image velocimetry (PIV) and measurements on the heated duct wall. They found that the synthetic jet tends to retard or block the duct flow while a 25 percent increase in thermal performance was observed.

Numerical studies on thermal performance of synthetic jet with cross flow interaction are also very limited in published literature. Such significant work is presented by Timchenko et al. [15] who investigated the use of a synthetic jet induced by a vibrating diaphragm to enhance the heat transfer in a $200 \mu \mathrm{m}$ microchannel. Their two-dimensional (2-D) transient simulation considered the jet acting in cross-flow to the bulk flow in the channel with the diaphragm assumed to execute a parabolic motion. They observed a 64 percent improvement in cooling at the impinging wall for the flow conditions used. Despite the recognised significance of flow turbulence in synthetic jet flows, their analysis however did not include an appropriate turbulence model in the simulation.

In a recent study, Erbas and Baysal [16] conducted computational work of a synthetic jet actuator in a two-dimensional channel to assess its thermal effectiveness on a heated surface protruding into the fluid as a step. They varied the number of actuators, placement and phasing of the membrane concluding that the heat transfer rate would increase with the number of jets, appropriate jet spacing, the use of nozzle-type orifice geometry and $180^{\circ}$ out of phase jet operation. However, the investigation did not examine the influence of cross flow on the thermal performance.

The present study examines a novel thermal enhancement strategy for micro-scale heat transfer applications by combining the benefits of highly favourable synthetic jet characteristics and the proven effectiveness of microchannel flows. This hybrid arrangement is envisaged to deliver excellent thermal performance for microchannel heat sinks without the need for additional fluid circuits and large fluid velocities that invariably increase pressure drop. The published literature reports a very few numerical analyses akin to highly interactive combined synthetic jet and microchannel flows considered in this paper while very limited applicable experimental work is available for comparison.

\section{NUMERICAL MODEL \\ 2.1 Governing Equations}

This study investigates the heat transfer characteristics of low Reynolds number turbulent synthetic jets operating in a confined region while interacting with microchannel flow. Applicable governing equations for the analysis are the Navier-Stokes equations, the continuity equation and the energy equation subject to applied boundary conditions. Air is assumed to be an incompressible Newtonian fluid although compressibility effects are considered later. In this, the equation of continuity is written as:

$$
\frac{\partial u}{\partial x}+\frac{\partial v}{\partial y}=0
$$

the momentum equations are expressed as:

$\mathrm{x}$-momentum

$$
\frac{\partial u}{\partial t}+u \frac{\partial u}{\partial x}+v \frac{\partial u}{\partial y}=-\frac{\partial p}{\partial x}+\mu\left(\frac{\partial^{2} u}{\partial x^{2}}+\frac{\partial^{2} u}{\partial y^{2}}\right)
$$

y-momentum

$$
\frac{\partial v}{\partial t}+u \frac{\partial v}{\partial x}+v \frac{\partial v}{\partial y}=-\frac{\partial p}{\partial y}+\mu\left(\frac{\partial^{2} v}{\partial x^{2}}+\frac{\partial^{2} v}{\partial y^{2}}\right)
$$

and the energy equation and its associated expressions are written as:

$$
\frac{\partial}{\partial t}(\rho E)=-\nabla \cdot(\mathbf{U}(\rho E+p))+k_{\text {eff }} \nabla^{2} T
$$


Where $\mathrm{k}_{\text {eff }}$ is the effective conductivity and $\mathrm{E}$ is the total energy given by,

$$
\mathrm{E}=\mathrm{h}-\frac{\mathrm{p}}{\rho}+\frac{\mathrm{U}^{2}}{2}
$$

The local Nusselt number $\mathrm{Nu}(\mathrm{x}, \mathrm{t})$ wherein the local wall heat transfer coefficient $\mathrm{h}$ is embedded, describes the convective heat transfer between the heated surface and the synthetic jet flow. Using orifice width $d_{o}$ as the characteristic length, this is defined as

$$
\mathrm{Nu}(\mathrm{x}, \mathrm{t})=\frac{\mathrm{hd}_{\mathrm{o}}}{\mathrm{k}_{\mathrm{f}}}=\frac{\partial \mathrm{T}}{\partial \mathrm{y}} \frac{\mathrm{d}_{\mathrm{o}}}{\Delta \mathrm{T}}
$$

The Nusselt number is evaluated from the local surface normal temperature gradient, $\frac{\partial T}{\partial y}$ and the temperature difference $\Delta T=\left(T_{w}-T_{b}\right)$ where $T_{w}$ is the local wall temperature and $T_{b}$ is the average bulk fluid temperature the in the fluid domain.

\subsection{Solution Domain and Boundary Conditions}

Similar to Fugal [17] and Wang [18], the present study uses a numerical model developed on the computational fluid dynamics software FLUENT. A structured mesh was developed for the solution domain shown in Figure 3 using the mesh generation facility GAMBIT. The grid dependency of results was tested by observing the changes to time-averaged velocity fields in the solution domain for varied grid sizes of 14000 (course), 48072 (medium) and 79000 (fine). In view of the moving mesh integrity and CPU time, the most appropriate grid size was found to be 48072 cells for a five percent tolerance between successive grid selections. In capturing intricate details of the jet formation and flow separation, the grid density in the vicinity of the orifice was refined to have 14 grid cells in the axial direction and 20 in the transverse direction.

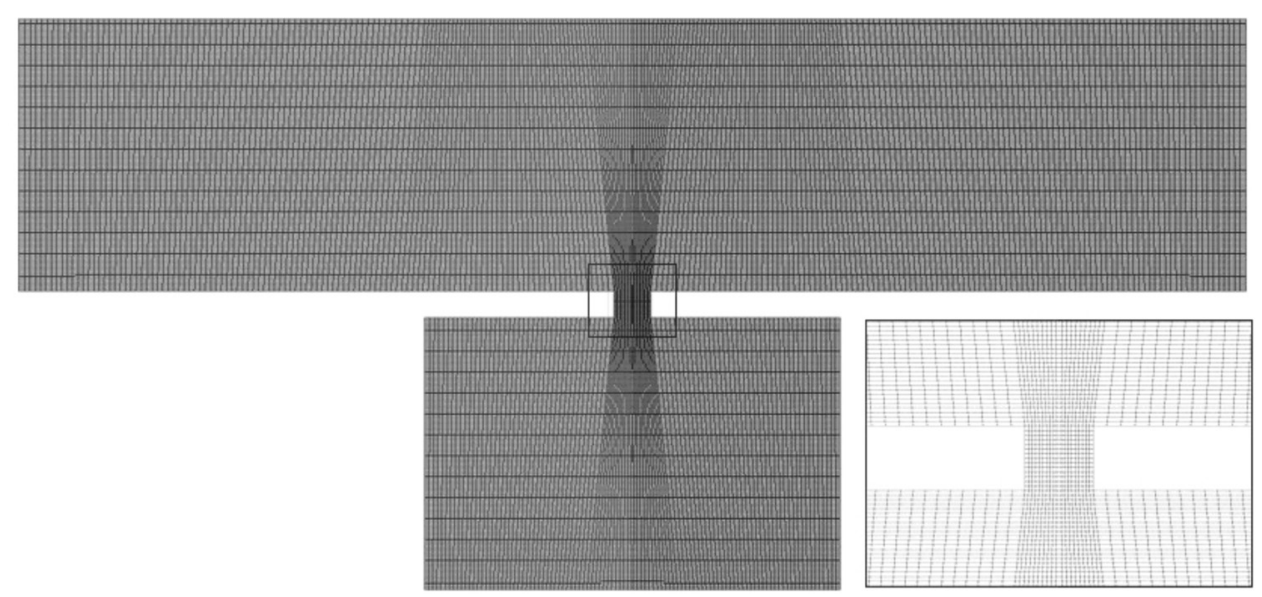

Figure 3. Computational grid for solution domain Inset shows enlarged view of the marked region.

As illustrated by Utturkar et al. [19], the vortex formation in synthetic jet is governed by the nondimensional groups Reynolds number $(\mathrm{Re})$ and Stokes number $(\mathrm{S})$, and occurs under the parametric condition of $\mathrm{Re} / \mathrm{S}^{2}>\mathrm{K}$, where the constant $\mathrm{K} \approx 1$ for two-dimensional jets and 0.16 for axi-symmetric synthetic jets. By due consideration of this requirement, the following dimensions were used in the analysis: orifice width $d_{o}=50 \mu \mathrm{m}$, orifice length $h_{o}=50 \mu \mathrm{m}$, channel height $\mathrm{H}=500 \mu \mathrm{m}$, channel length $\mathrm{D}=2250 \mu \mathrm{m}$, heater length $\mathrm{L}=750 \mu \mathrm{m}$, cavity width $d_{c}=750 \mu \mathrm{m}$ and cavity height $\mathrm{h}_{c}=500 \mu \mathrm{m}$. This dimensional selection was checked and confirmed for its compliance with the continuum mechanics for the scale of the attempted problem using Knudsen number Kn, which is the ratio of the molecular free path length to representative length. 
Adiabatic conditions were applied at the cavity walls and the diaphragm while the heater surface was maintained at an isothermal temperature of $360 \mathrm{~K}$. The (left) flow inlet to the microchannel was treated as a known constant velocity boundary while the (right) flow outlet was treated as pressure outlet boundary. It was assumed that the working fluid air is incompressible and has an inlet temperature of $300 \mathrm{~K}$ with constant thermodynamic properties under standard atmospheric conditions.

Arising from small geometrical length scales, synthetic jets generally tend to have small operating Reynolds numbers making flow turbulence seemingly unimportant. However, the oscillating nature of the flow may give rise to intense localised perturbations. In handling the wide flow variations, the Shear-Stress-Transport (SST) k- $\omega$ turbulence model was invoked in the model to provide an accurate representation of the near-wall region of wall-bounded turbulent flows. Initially $3 \%$ turbulence intensity is applied at the outlets, thereafter the flow is allowed to develop on its own through the inherent instabilities. The $\mathrm{y}+$ and $\mathrm{y}^{*}$ value in the wall region were found to be approximately 1 , confirming that the near-wall mesh resolution is in the laminar sublayer.

\subsection{Initial Conditions and Solution Methodology}

The initial $(t=0)$ position of the diaphragm was taken to be at the bottom of the cavity. A special User Defined Function (UDF) incorporating Dynamic-layering technique [20] was formulated and combined with the FLUENT solver to describe the periodic diaphragm movement. For this, the diaphragm displacement was expressed as $\mathrm{y}=\mathrm{A} \sin (\omega \mathrm{t})$, where $\mathrm{A}$ is the diaphragm amplitude, $\omega$ is the angular frequency and $t$ is time.

A segregated solution method with implicit solver formulation in FLUENT was used as the numerical algorithm while the Second-order discretisation schemes were employed for density, momentum, pressure, kinetic energy, specific dissipation rate and energy. The Pressure-Implicit with Splitting of Operators (PISO) scheme was used for pressure-velocity coupling. The bulk temperature of air at every time step was calculated using an UDF while the updated bulk temperature was fed back to the simulation for calculating local heat transfer coefficient and Nusselt number.

The jet Reynolds number $\left(\operatorname{Re}_{c}\right)$ was calculated based on the jet characteristic velocity $U_{c}$, which is defined by Smith [21] as,

$$
\mathrm{U}_{\mathrm{c}}=\mathrm{L}_{\mathrm{s}} \mathrm{f}=\frac{1}{\mathrm{~T}} \int_{0}^{\mathrm{T} / 2} \mathrm{u}_{0}(\mathrm{t}) \mathrm{dt}
$$

where $u_{o}(t)$ is the jet velocity at the orifice discharge plane, $1 / 2 T$ is the jet discharge time or half period of diaphragm motion, and $\mathrm{L}_{\mathrm{s}}$ is the stroke length (defined as the discharged fluid length through orifice during the upward diaphragm stroke).

With these imposed conditions, the unsteady, Reynolds-averaged Navier-Stokes equations within the solution domain were solved along with the energy equation for a range of operating conditions, which are given in Table 1.

Table 1: Parametric range for numerical simulation

\begin{tabular}{lc}
\hline Parameter & Range \\
\hline Microchannel inlet velocity, $\mathrm{V}_{\mathrm{i}}(\mathrm{m} / \mathrm{s})$ & $0,0.5,1.0,2.0$ \\
Diaphragm frequency, $\mathrm{f}(\mathrm{kHz})$ & 10 \\
Diaphragm Amplitude, $\mathrm{A}(\mu \mathrm{m})$ & $0,25,50,75,100$ \\
Jet Reynolds Number, $\mathrm{Re}_{\mathrm{c}}$ & $15,30,46,62$ \\
Distance from orifice to heated wall, $\mathrm{H} / \mathrm{d}_{\mathrm{o}}$ & 10 \\
\hline
\end{tabular}

The simulation was carried out using 720 time steps per cycle wherein 20 sub-iterations were performed within each time step. At each time step of a cycle, the internal iterations were continued until the residuals of mass, momentum, turbulence parameters $\left(\mathrm{k}\right.$ and $\omega$ ) were reduced below $10^{-3}$ and energy residuals were reduced below $10^{-6}$, which is the convergence criterion for the computation. Data were extracted at every twentieth time step giving 36 data points per cycle. It was observed that 10 diaphragm cycles would be sufficient to achieve quasi-steady operating conditions in this microchannel flow geometry. To increase accuracy, a 2D-double precision solver was used to solve the governing equations for heat and fluid flow. 


\section{RESULTS AND DISCUSSION}

\subsection{Model Validation}

The model validation for the present simulation was carried out by developing a separate synthetic jet model by altering the micro-scale domain dimensions to match the published work involving miniscale geometries of Yao et al. [22]. These results were extensively incorporated in NASA Langley Research Centre Workshop (CFDVAL2004) [23] in assessing suitability of turbulence models for synthetic jet flows. The results of the workshop indicated that the turbulence model selected significantly affected the results and Shear-Stress-Transport (SST) k- $\omega$ turbulence model works best among the URANS models.

The predicted axial (y-velocity) was compared with the experimental jet velocities measured by Yao et al. [22] using the techniques of Particle Image Velocimetry (PIV), Hot wire anemometry and Laser Doppler Velocimetry (LDV). The present simulation agreed very well with the experimental data validating the model and its accuracy. The details of this validation and grid independence studies are further discussed in Jagannatha et. al. [24, 25].

\subsection{Velocity and Fluid Flow Characteristics}

For an operating frequency of $10 \mathrm{kHz}$ and diaphragm amplitude of $25 \mu \mathrm{m}$, Figure 4 illustrates the diaphragm displacement and the jet discharge velocity over one full cycle completed in 0.1 milliseconds. The jet velocity fluctuation resembling a sinusoidal pattern clearly indicates zero net discharge of fluid mass through the orifice, as anticipated in synthetic jet operation.

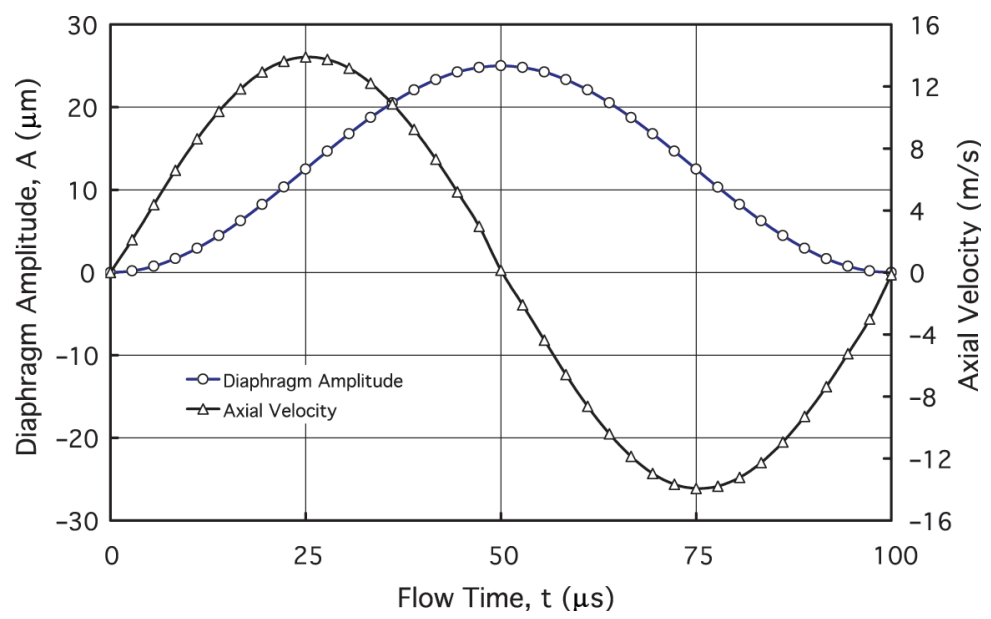

Figure 4. Diaphragm displacement and jet velocity over one complete cycle at $\mathrm{A}=25 \mu \mathrm{m}$ and $\mathrm{f}=10 \mathrm{kHz}$.

Figures 5 (a) and 5 (b) show typical time-lapsed velocity contours within the solution domain respectively for two separate cases of synthetic jet interacting with stagnant fluid as well as flowing fluid in the micro passage.

During the diaphragm upward motion, a high-velocity fluid jet is discharged through the cavity orifice into the flow in micro passage. Determined by diaphragm amplitude, sufficiently strong jet momentum enables the jet to penetrate the micro passage flow to reach the heated (upper) wall within the time up to $t=1 / 2 \mathrm{~T}$ at the peak diaphragm displacement.

In the figures, the formation of synthetic jet vortices is clearly visible during this initial phase of sequence. The flow patterns exhibits symmetry in Figure 5 (a) while the cross-flow drag imparted by the micro passage fluid stream gives rise to asymmetry in Figure 5 (b) where the jet is swayed in the streamwise direction.

For $t>1 / 2 T$, the diaphragm retreats from its peak displacement to complete the cycle. During this final phase, the synthetic jet mechanism draws fluid back into the cavity. Meanwhile, the synthetic jet vortices formed previously are dragged downstream by the micro passage flow. 

Microchannel with Cross-flow Synthetic Jet

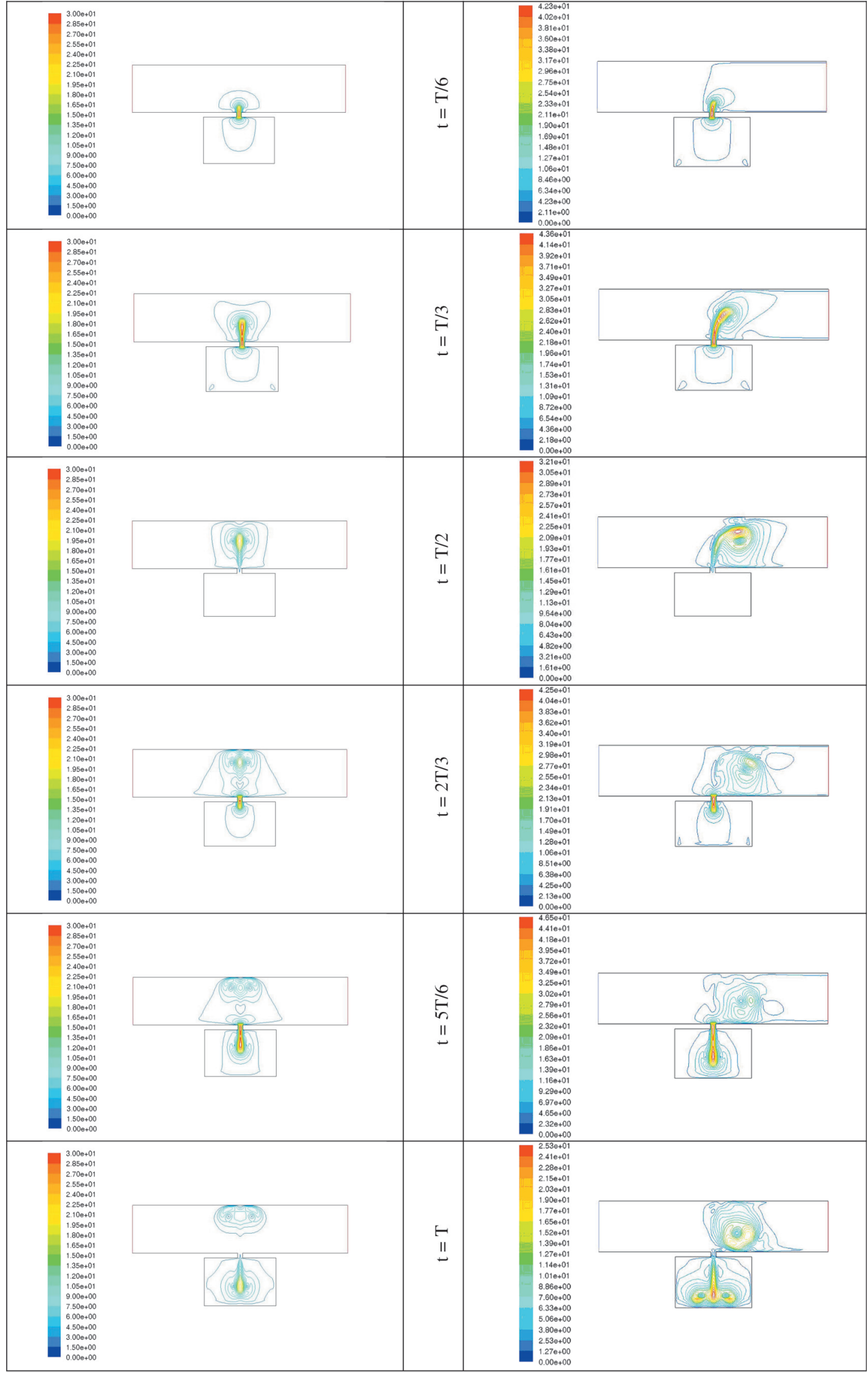

(a) $\mathrm{V}_{\mathrm{i}}=0 \mathrm{~m} / \mathrm{s} \mathrm{A}=50 \mu \mathrm{m}$ and $\mathrm{f}=10 \mathrm{kHz}$

(b) $\mathrm{V}_{\mathrm{i}}=0.5 \mathrm{~m} / \mathrm{s} \mathrm{A}=75 \mu \mathrm{m}$ and $\mathrm{f}=10 \mathrm{kHz}$

Figure 5. Time-lapsed velocity contours over one diaphragm cycle. 
The examination of these figures reveals that in spite of the channel fluid flow, the synthetic jet still carries all of its fundamental characteristics observed under stagnant flow conditions. The synthetic jet action periodically interrupts the microchannel flow and breaks up the developing thermal and hydrodynamic boundary layers at the heated top wall. This cross-flow interaction creates steep velocity and temperature gradients at the heated surface as long as jet impingement occurs. This pulsating flow mechanism therefore leads to improved thermal characteristics in the synthetic jet-mounted microchannel arrangement.

The degree of skewness in the jet discharge velocity profile is envisaged to be a prime factor determining the synthetic jet vortex formation process at the orifice. Vindicating evidence for this in the presence of channel cross-flow is depicted in Figure 6 that shows the velocity vectors near jet orifice at $\mathrm{t}=\mathrm{T} / 2$ for $\mathrm{V}_{\mathrm{i}}=0.5 \mathrm{~m} / \mathrm{s}$ and $2.0 \mathrm{~m} / \mathrm{s}$. It is clearly noticed that, higher channel flow causes increased fluid recirculation or entrainment in the orifice passage and its vicinity. Hence, favourable flow conditions are created for more vigorous synthetic jet vortices because the fluid recirculation is an identified key parameter that decides vortex formation.

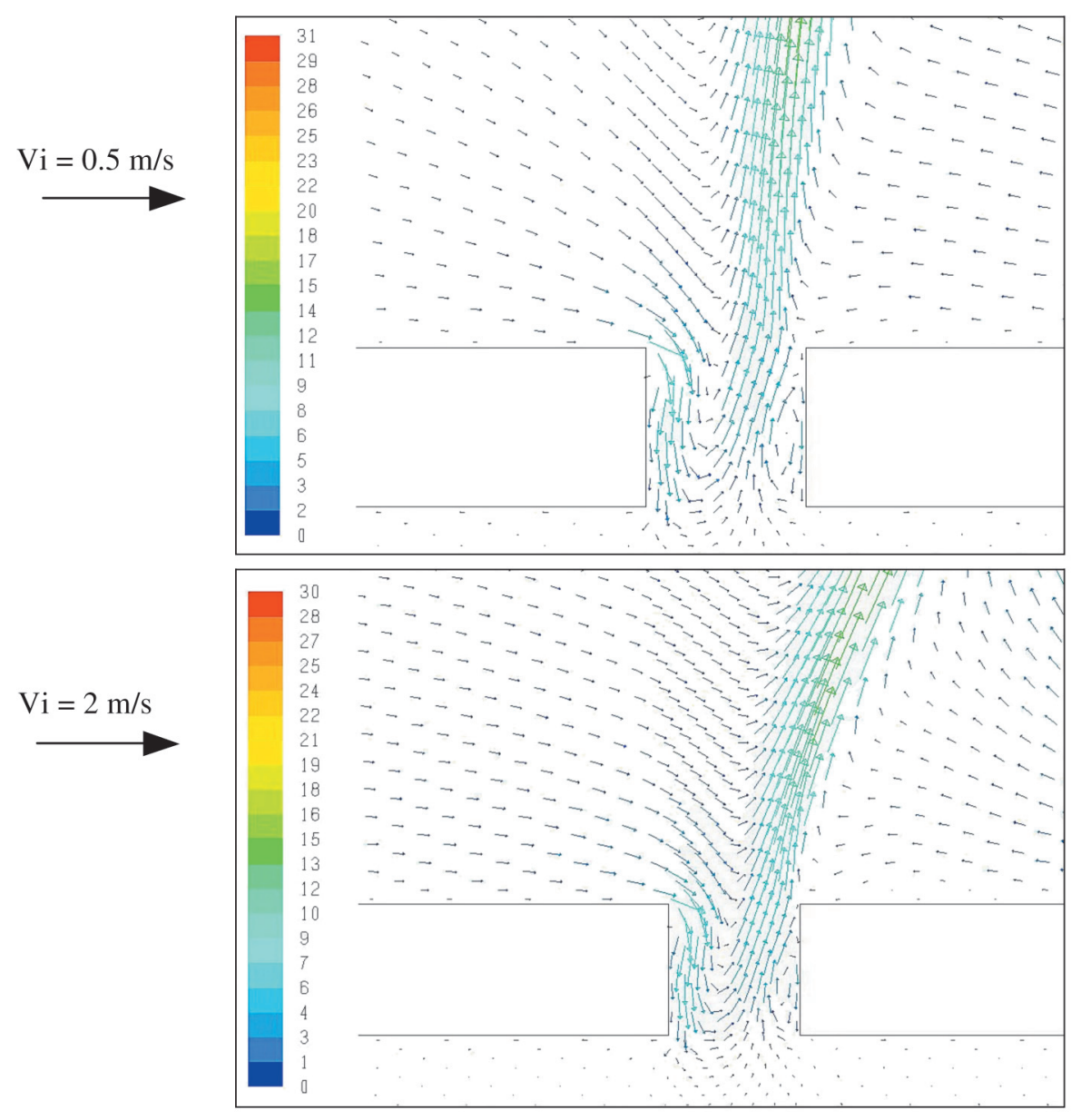

Note: Length of arrows and colours indicate velocity magnitude $(\mathrm{m} / \mathrm{s})$

Figure 6. Velocity vectors near the orifice at time $t=T / 2$ (maximum expulsion)

$$
f=10 \mathrm{kHz}, \mathrm{A}=50 \mu \mathrm{m} \text {. }
$$

Additional insight on synthetic jet penetration through the cross flow is gained from Figure 7 that presents the jet propagation velocity at several chosen planes parallel to the orifice. These plots are obtained for the maximum diaphragm displacement that occurs at $\mathrm{t}=\mathrm{T} / 2$ with $\mathrm{f}=10 \mathrm{kHz}$ and $\mathrm{A}=50 \mu \mathrm{m}$. Corresponding velocity profiles for a synthetic jet in stagnant fluid medium are also shown for comparison.

This series of figures shows that, compared to the stagnant fluid case, the peak axial velocity at each location is swept downstream and somewhat attenuated with the increased cross-flow velocity. A reduction in axial velocity impedes the jet's boundary layer penetrating ability resulting in diminished thermal performance. 

Microchannel with Cross-flow Synthetic Jet
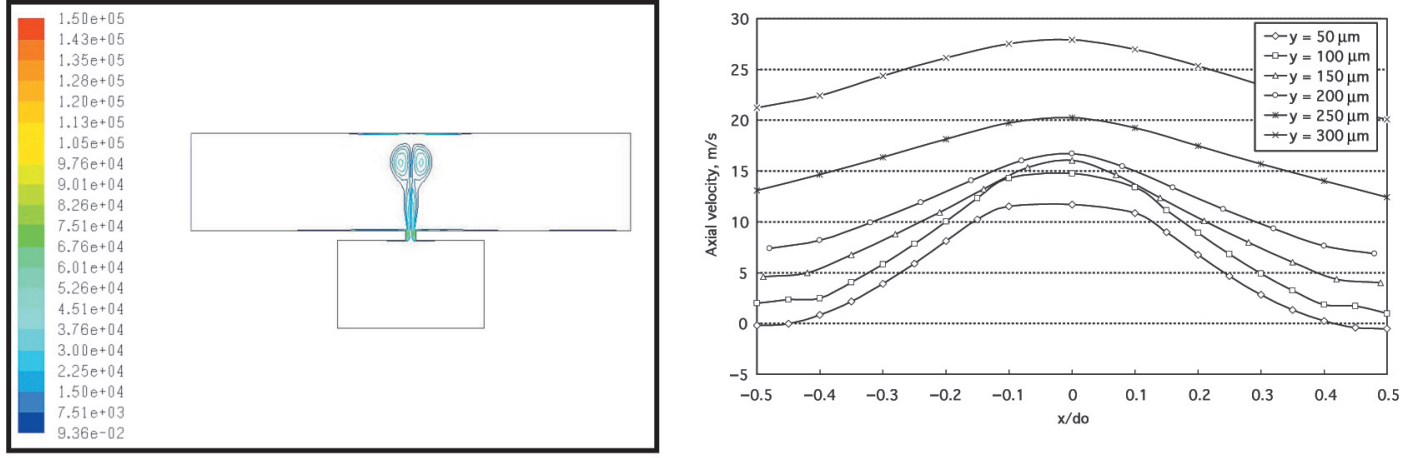

(a) $\mathrm{V}_{\mathrm{i}}=0 \mathrm{~m} / \mathrm{s}$ (stagnant fluid)
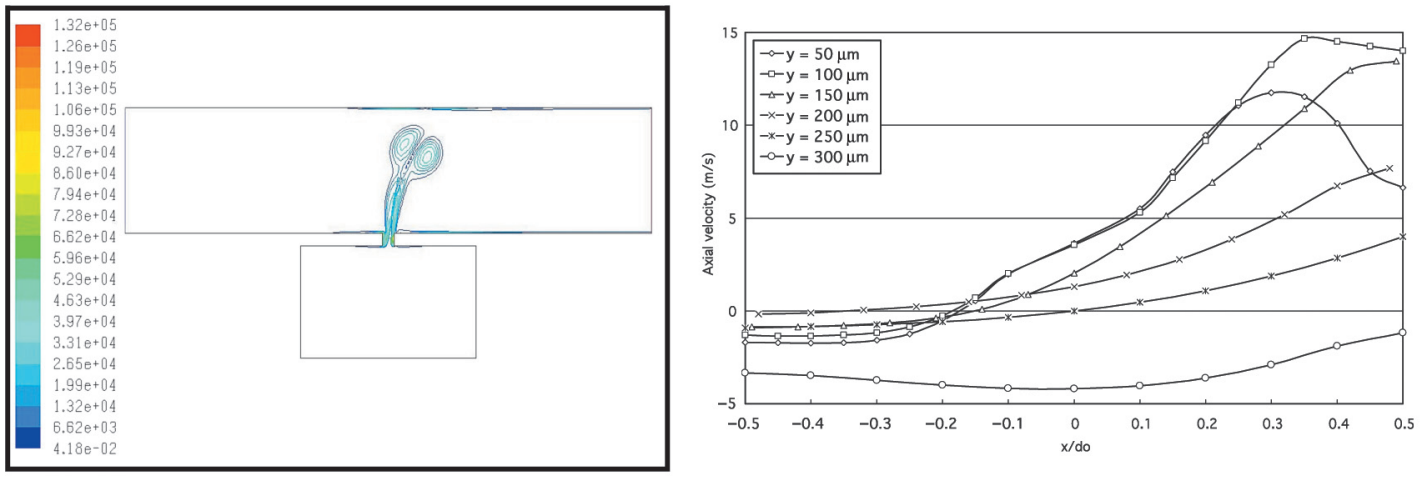

(b) $\mathrm{V}_{\mathrm{i}}=0.5 \mathrm{~m} / \mathrm{s}$
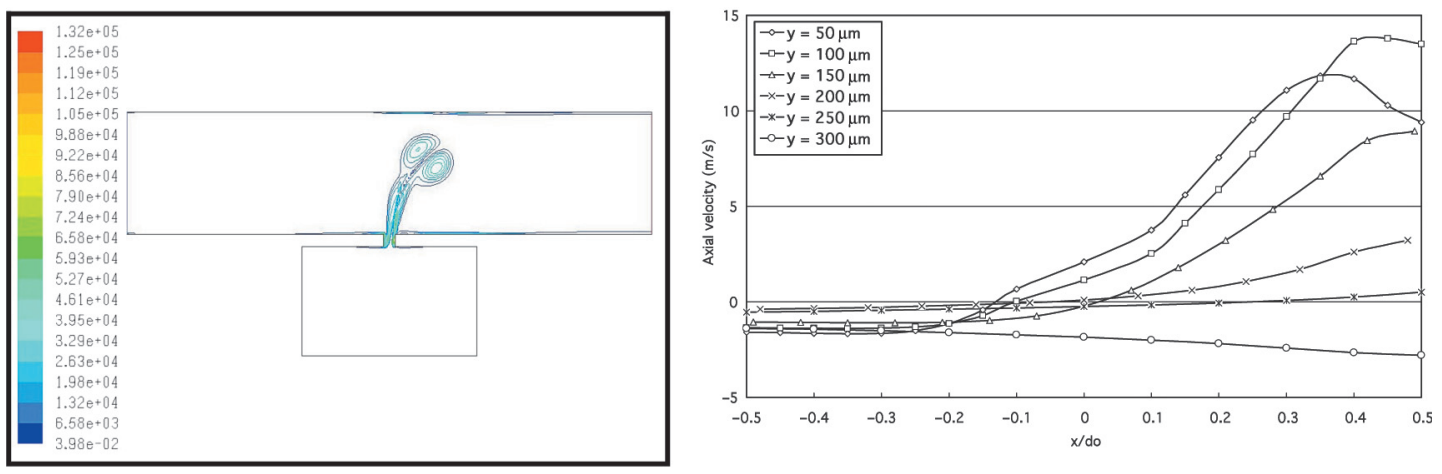

(c) $\mathrm{V}_{\mathrm{i}}=1.0 \mathrm{~m} / \mathrm{s}$
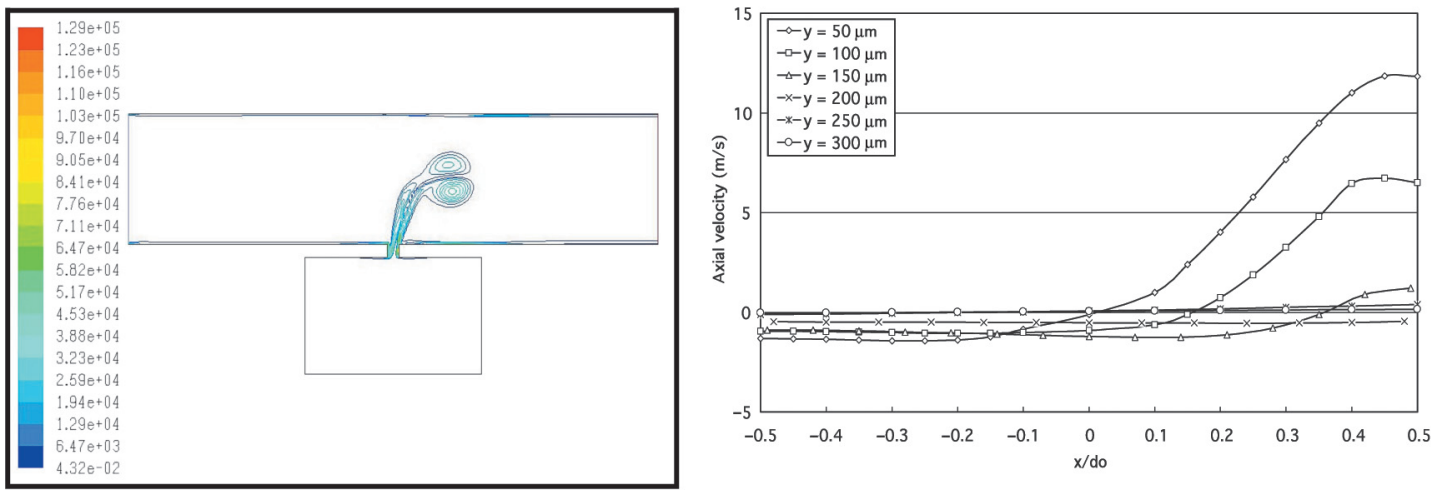

(d) $\mathrm{V}_{\mathrm{i}}=2.0 \mathrm{~m} / \mathrm{s}$

Figure 7. Effect of cross-flow on jet propagation in the microchannel at $\mathrm{f}=10 \mathrm{kHz}$ and $A=50 \mu \mathrm{m}$. 


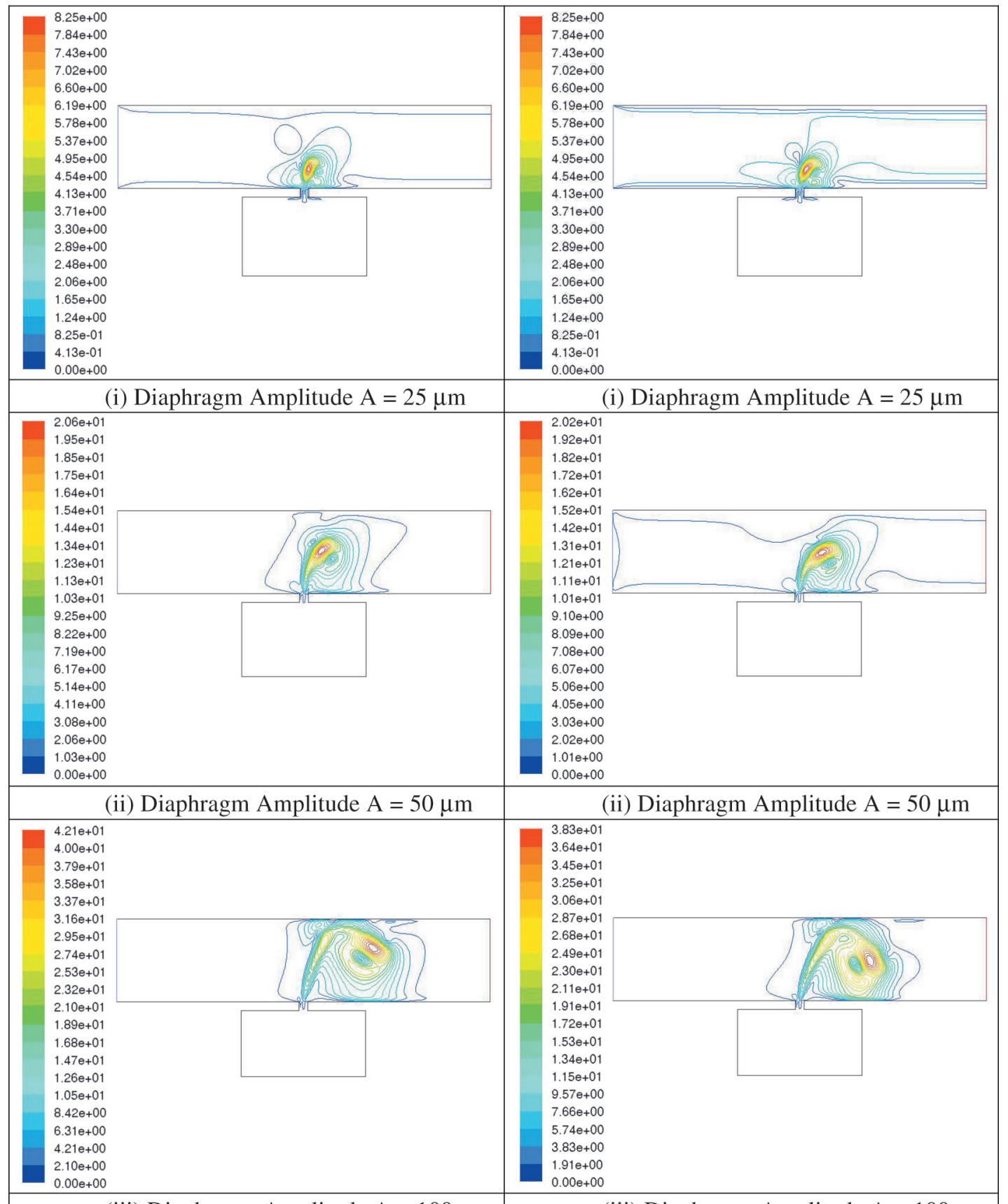

(iii) Diaphragm Amplitude $\mathrm{A}=100 \mu \mathrm{m}$

(iii) Diaphragm Amplitude $\mathrm{A}=100 \mu \mathrm{m}$

(a) $\mathrm{V}_{\mathrm{i}}=0.5 \mathrm{~m} / \mathrm{s}, \mathrm{f}=10 \mathrm{kHz}$

(b) $\mathrm{V}_{\mathrm{i}}=1.0 \mathrm{~m} / \mathrm{s}, \mathrm{f}=10 \mathrm{kHz}$

Figure 8. Effect of diaphragm amplitude illustrated by velocity contours at $t=1 / 2 \mathrm{~T}$.

Relative strengths of the synthetic jet and the microchannel flow drag determine the extent of cross-flow interference and the boundary layer disruption at the heated wall. This is illustrated in Figures 8 (a) and 8 (b) for microchannel velocities of $0.5 \mathrm{~m} / \mathrm{s}$ and $1.0 \mathrm{~m} / \mathrm{s}$. It is clearly evident that the increased microchannel flow causes the jet to be swayed downstream. This impedes the jet's ability to penetrate through the boundary layer to reach the heated wall to bring about favourable thermal characteristics.

\subsection{Thermal Characteristics and Heat Transfer Enhancement}

Through its unique vortex generation process, the proposed synthetic jet mechanism creates in the channel, fluid flow structures that are very conducive for wall heat transfer. Comparing its performance against a 
conventional technique, this section evaluates the degree of thermal enhancement when the synthetic jet arrangement is integrated into a flow channel in view of potential for novel heat sink development.

For thermal performance comparison, a new simulation is developed by some modifications to the standard synthetic jet model with cross-flow described in Figure 2. In this, the analysis is now performed without actuating the periodic diaphragm movement to stop the jet discharge into the channel fluid stream while retaining all the other geometrical and boundary conditions as for the standard synthetic jet model with cross-flow [Figure 2]. In the absence of synthetic jet operation, this new simulation is simplified and forms a case of pure forced convection in the channel.

Figure 9 shows the fluid temperature contours obtained by the modified channel flow simulation. It is clearly seen that the thermal boundary layer is getting thinner with increased channel inlet velocities, therefore improving channel heat transfer. For selected channel velocities, Figure 10 depicts the local Nusselt number variation over the heated wall in the channel without synthetic jet operation. It is readily identified that this distribution is similar to that of flow over a flat plate, and the Nusselt number asymptotically decays from the leading edge of heated wall with the developing flow.

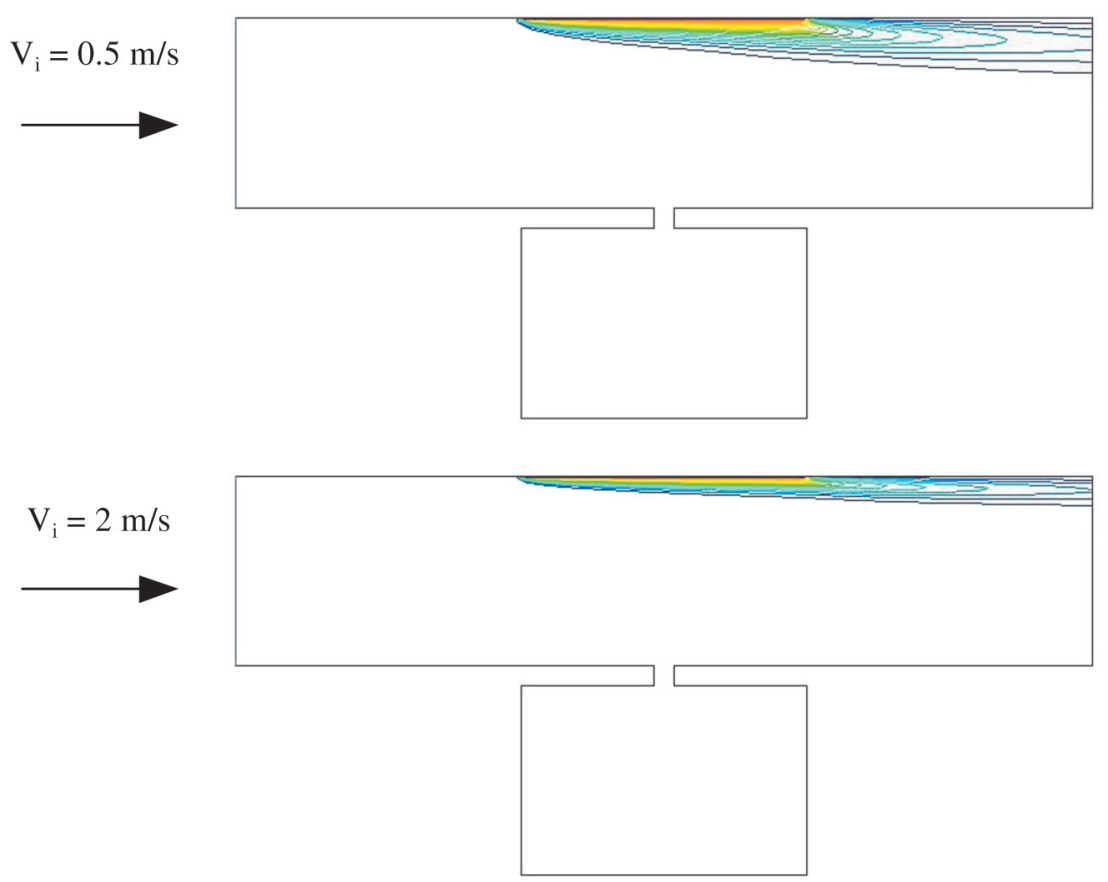

Figure 9. Temperature contours in channel flow without synthetic jet operation.

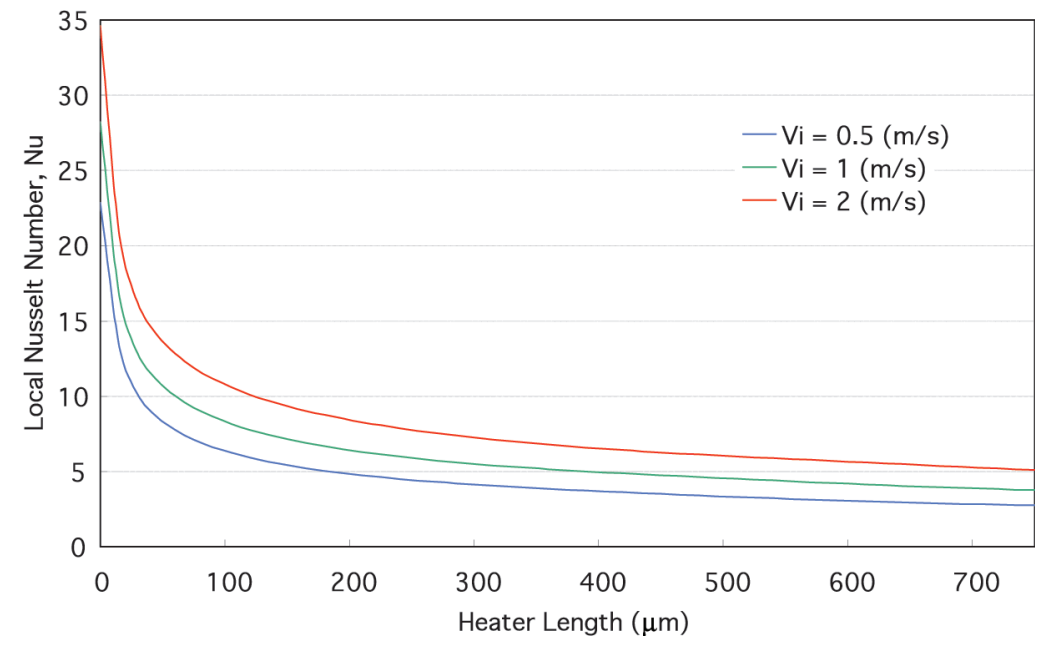

Figure 10. Variation of Nusselt number along the heater surface with channel velocity. 
For a typical case with stagnant fluid $\left(\mathrm{V}_{\mathrm{i}}=0 \mathrm{~m} / \mathrm{s}\right)$ in the microchannel, Figure 11 shows the distribution of local Nusselt numbers over the heated wall for several time steps during one cycle of operation. It indicates that initially, the Nusselt number remains very low for $0<1 / 2 \mathrm{~T}$. During this period, the synthetic jet vortices have yet to impinge on the heated surface. The Nusselt number very rapidly increases to about 23 for $\mathrm{t}=2 \mathrm{~T} / 3$ when the vortices begin to interact with the heated surface. A gentle decline in the Nusselt number is then noticed for $\mathrm{t}>2 \mathrm{~T} / 3$.

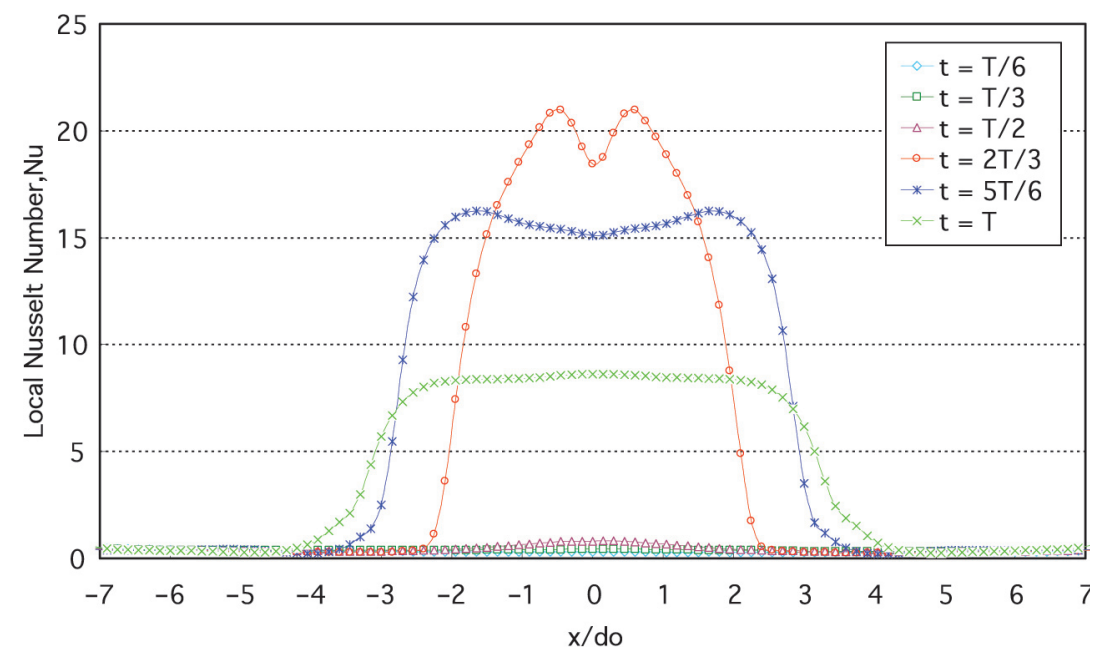

Figure 11. Distribution of local nusselt number at the heated wall over one cycle $\mathrm{f}=10 \mathrm{kHz}, \mathrm{A}=50 \mu \mathrm{m}$ and $\mathrm{V}_{\mathrm{i}}=0 \mathrm{~m} / \mathrm{s}$.

Figure 12 shows a typical distribution of local Nusselt numbers at the heated wall over one cycle for the case of fluid flowing $\left(\mathrm{V}_{\mathrm{i}}=1 \mathrm{~m} / \mathrm{s}\right)$ in the microchannel. It is noticed that the distribution now shifted downstream and the peak value of the Nusselt number is reduced to about 13. This is because the increased microchannel flow interacts with the impinging jet and drags it with the flow, as depicted in Figures 7 and 8 . Thus, the velocity and temperature gradients at the heated wall are reduced along with the heat transfer rates.

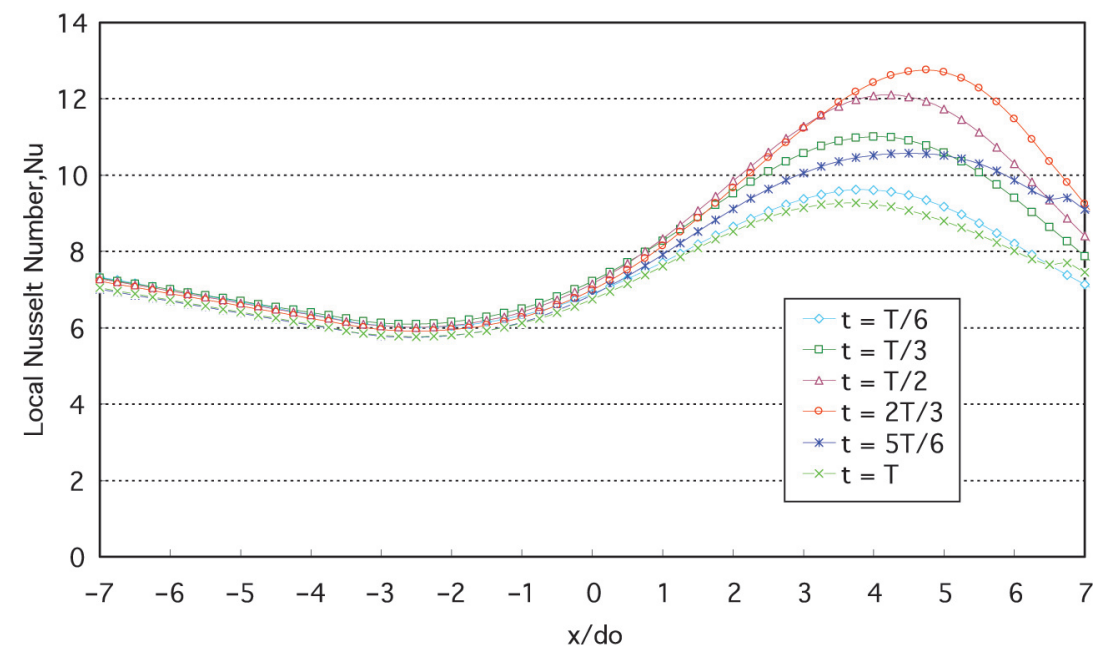

Figure 12. Distribution of local Nusselt number at the heated wall over one cycle $\mathrm{f}=10 \mathrm{kHz}, \mathrm{A}=50 \mu \mathrm{m}$ and $\mathrm{V}_{\mathrm{i}}=1 \mathrm{~m} / \mathrm{s}$.

Figure 13 depicts the degree of thermal enhancement achieved by the introduction of a cross-flow pulsating synthetic jet for selected microchannel flow velocities. In this, the thermal performance of microchannel flow without the cross-flow jet mechanism is taken as the datum, which represents the value of 1.0 at zero jet Reynolds number. For the tested range of diaphragm amplitude, jet frequency and 


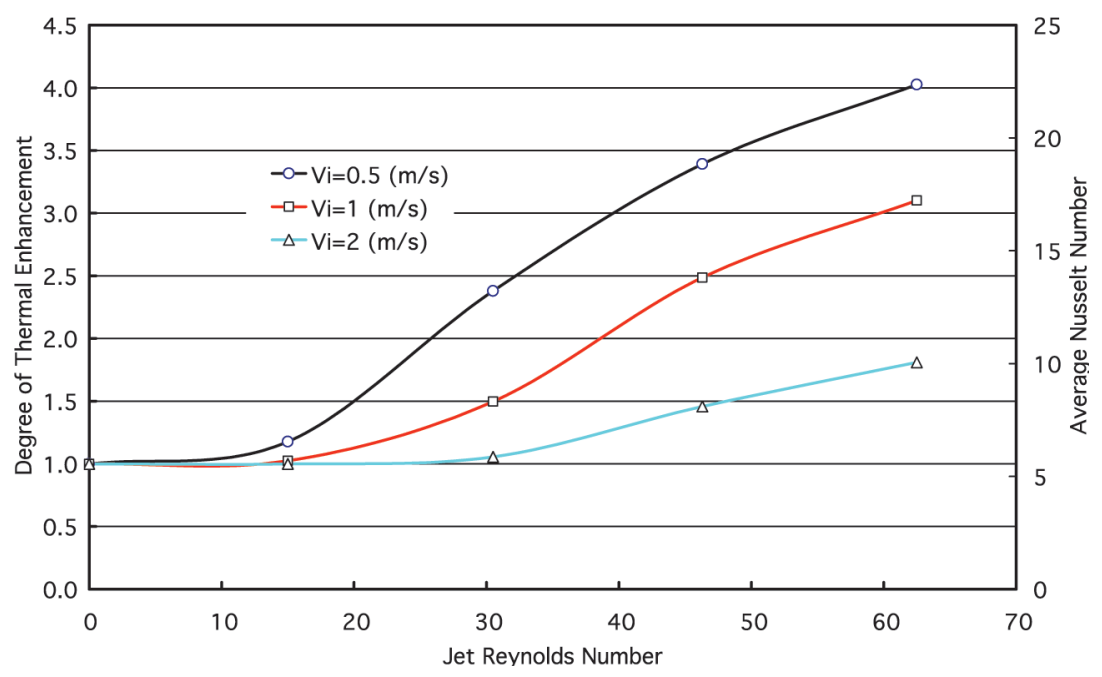

Figure 13. Degree of thermal enhancement and Nusselt number with synthetic jet mechanism.

microchannel velocity, the synthetic jet mechanism delivers about 4.3 times thermal enhancement in the microchannel compared to a channel without pulsating jet. Because of the nature of synthetic jet mechanism, this degree of thermal enhancement is realised without introducing additional net mass flow into the microchannel or requiring additional fluid circuits, which is recognised as a major operational benefit.

Figure 13 further shows that the higher microchannel velocity impairs thermal enhancement level. As explained previously with reference to Figures 7 and 8, the reason for this reduced performance is because the drag of microchannel flow swaying the synthetic jet downstream preventing jet impingement on the heated surface. As the jet Reynolds number (or jet velocity or diaphragm amplitude) is increased for a fixed microchannel velocity, the degree of thermal enhancement suddenly increases when the jet is able to penetrate and reach the heated surface. In Figure 13, this situation is shown by the onset of sharp elevation in thermal enhancement gradient at a jet Reynolds number of approximately 15 . As the microchannel velocity is increased, this point of rapid growth tends to shift towards higher values of jet Reynolds number.

Figure 14 shows the thermal performance and the pressure drop of microchannel flow without synthetic jet mechanism. It is seen for example that, to obtain 4.3 times thermal enhancement possible with the hybrid microchannel having flow velocity of $0.5 \mathrm{~m} / \mathrm{s}$, a channel without the pulsed jet will need 40 times velocity increase. Such a channel will have very adverse 70 -fold increase in flow pressure drop. This clearly demonstrates the significant potential for thermal enhancement in microchannels with this hybrid arrangement and its operational benefits as an enhancement technique.

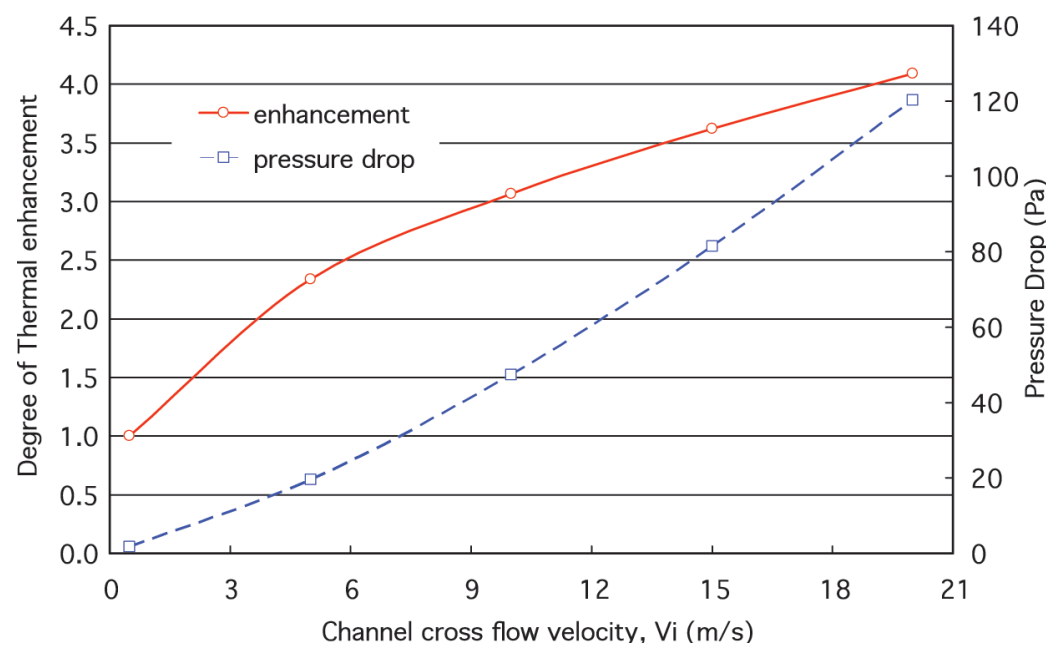

Figure 14. Degree of thermal enhancement and pressure drop without synthetic jet mechanism. 


\subsection{Influence of Fluid Compressibility on Synthetic Jet Operation}

The high frequency operation of the diaphragm may render the compressibility of the working fluid (air) to be a significant factor in determining the thermal performance of the microchannel-synthetic jet arrangement. This influence is examined by conducting two identical simulations. The first analysis is performed assuming constant air density, so that the fluid compressibility is totally eliminated. For comparison, the fluid density variations with local pressure within the solution domain are considered in the second simulation.

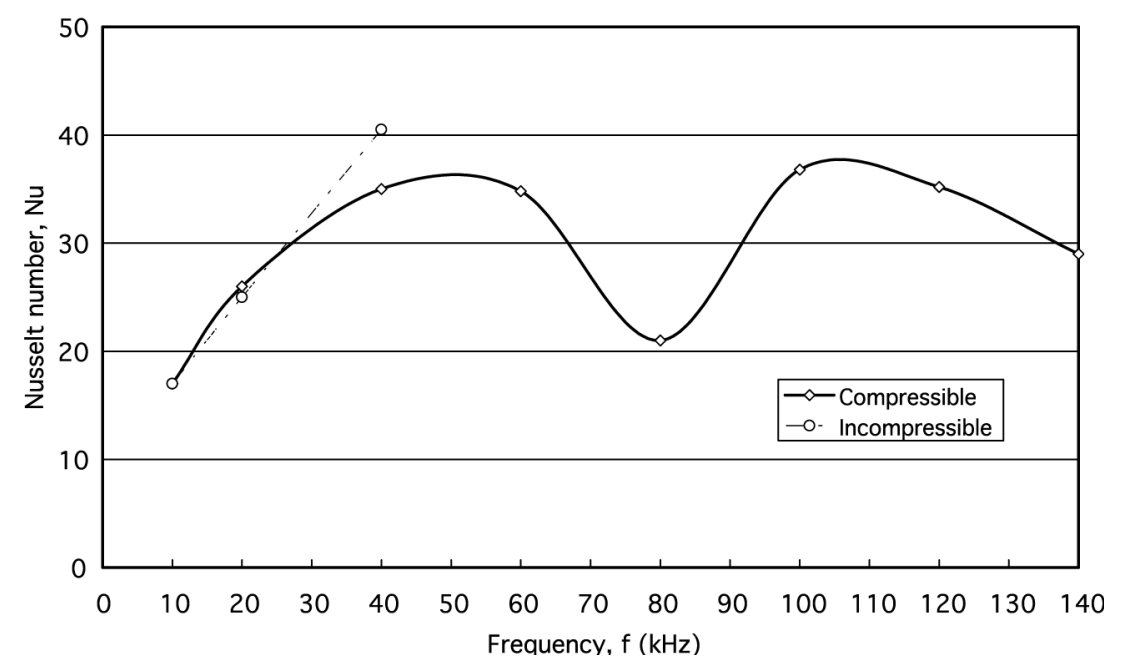

Figure 15. Effect of compressibility on the heat transfer characteristic.

Figure 15 illustrates the variation of Nusselt number against diaphragm frequency for the two analyses with and without air compressibility. The Nusselt number for both cases is seen to closely follow each other while increasing almost linearly up to about a frequency of $20 \mathrm{kHz}$. Beyond this value, the Nusselt number with compressibility accounted for suddenly deviates and indicates a marked fall in magnitude while the Nusselt number with incompressible flow conditions continues to grow. The former behaviour shows a minimum at a frequency of around $80 \mathrm{kHz}$. The simulation indicates that at this operating frequency, the fluid discharge through the orifice is almost ceased, which is attributed to the simultaneous expulsion and ingestion of air neutralising the flow though the orifice or formation of standing waves. Therefore, this condition imposes a temporary breakdown in the synthetic jet thermal effectiveness. The performance then quickly recovers beyond this critical diaphragm frequency. It is noted that this limiting operating frequency is closely correlated with the natural frequency of the cavity, which is obtainable as Slat Helmholtz frequency computed by,

$$
\mathrm{f}_{\mathrm{n}}=\frac{\mathrm{c}}{2 \pi} \sqrt{\frac{\mathrm{d}_{\mathrm{o}}}{\left(\left(\mathrm{h}_{\mathrm{o}} \times \mathrm{h}_{\mathrm{c}}\right) \times\left(\mathrm{d}_{\mathrm{o}}+\mathrm{d}_{\mathrm{c}}\right)\right)}}
$$

\section{CONCLUSIONS}

This study has successfully developed a numerical simulation to characterise the operation of a novel thermal enhancement technique for applications in microchannel heat sinks. The proposed mechanism utilises a pulsating fluid jet called "Synthetic Jet" that injects net positive fluid momentum with zero averaged fluid discharge into the microchannel. The pulsed interacts with the steady microchannel flow permeating for creating excellent thermal characteristics at the heated wall. In the tested range, this combined pulsed jet-microchannel arrangement delivers up to 4.3 times more wall heat transfer compared to a microchannel without the synthetic jet interaction. Under incompressibility conditions, the thermal performance continues to grow with the increased diaphragm frequency. When the fluid compressibility is accounted for, the thermal performance 
temporarily declines upon reaching a certain frequency and then recovers with further increase in its value. This hybrid flow system has a unique ability to achieve excellent heat transfer rates without higher microchannel flow velocities or causing pressure drop increase in flow passages. Also, this mechanism does not require the deployment of additional fluid flow circuits to achieve such high heat transfer rates. These are identified as key operational attributes that set it apart from other thermal enhancement strategies.

\section{REFERENCES}

[1] Lee P.S., Garimella S.V. and Liu D., Investigation of Heat Transfer in Rectangular Microchannels, International Journal of Heat and Mass Transfer, 2005,vol. 48(9), pp. 1688-1704.

[2] Qu W. and Mudawar I., Experimental and Numerical Study of Pressure Drop and Heat Transfer in a Single Phase Microchannel Heat Sink, International Journal of Heat and Mass Transfer, 2002, vol. 45(2), pp. 2549-2565.

[3] Lee P.S. and Garimella S.V., Thermally Developing Flow and Heat Transfer in Rectangular Mircochannels, International Journal of Heat and Mass Transfer, 2006, vol. 49(17), pp. 3060-3067.

[4] Steinke M.E. and Kandlikar S.G., Single Phase Heat Transfer Enhancement Technique in Microchannel and Minichannel Flows, International conference of Microchannel and Minichannels, ICMM 2004-2328, pp.141-148.

[5] Campbell J.S., Black W.Z., Glezer A., Thermal Management of a laptop Computer with Synthetic Air Microjets, InterSociety Conference on Thermal Phenomena, IEEE, 1998, pp. 43-50.

[6] Mahalingam R. and Glezer A., Air cooled heat sinks integrated with synthetic jets, InterSociety Conference on Thermal Phenomena, IEEE, 2002, pp. 285-291.

[7] Mahalingam R. and Rumigny N., Thermal Management using synthetic jet ejectors. IEEE, 2004, 27 (3), pp. 439-444.

[8] Gillespie M.B., Black W.Z., Rinehart C., and Glezer A., Local Convective Heat Transfer From a Constant Heat Flux Flat Plate Cooled by Synthetic Air Jets, Journal of Heat Transfer, 2006, 128, pp. 990-1000.

[9] Pavlova A. and Amitay M., Electronic Cooling Using Synthetic Jet Impingement, Journal of Heat Transfer, 2006, 128(9), pp. 897-907.

[10] Gilarranz J.L, Traub L.W and Rediniotis O.K. A new class of synthetic jet actuators-Part II: Application to flow separation control. Journal of Fluids Engineering, 2005,127, pp. 377-387.

[11] You D. and Moin P. Active control of flow separation over an airfoil using synthetic jets. Journal of Fluids and Structures, 2008, 24, pp. 1349-1357.

[12] Jabbal M. and Zhong S., The near wall effect of synthetic jets in a boundary layer. International Journal of Heat and Fluid flow, 2007, 29, pp. 119-130.

[13] Utturkar Y., Arik M. and Gursoy M., Assessment of cooling enhancement of synthetic jet in conjunction with forced convection, Proceedings of IMECE2007, Seattle, USA, November 11-15, 2007.

[14] Go D.B. and Mongia R.K., Experimental studies on synthetic jet cooling enhancement for portable platforms. IEEE, 2008, pp. 528-536.

[15] Timchenko V., Reizes J. and Leonardi E., A numerical study of enhanced micro-channel cooling using a synthetic jet actuator. Proceedings of the 15th Australasian Fluid Mechanics Conference, Sydney, Australia, 2004.

[16] Erbas N. and Baysal O., Micron-level actuators for thermal management of microelectronic devices, Heat Transfer Engineering, 2009, 30(1-2), pp. 138-147.

[17] Fugal S.R. and Smith B.L., A Numerical study of 2-D Synthetic jet formation, ASME Heat Transfer/Fluids Engineering Summer conference, ASME, Charlotte USA, 2004, pp 1-6.

[18] Wang Y., Yuan G. and Bidstrup S.A., Large eddy simulation (LES) for synthetic jet thermal management. International Journal of Heat and Mass Transfer, 2006, 49 (13-14), pp. 2173-2179.

[19] Utturkar Y. and Holman R., Formation Criterion for synthetic jets. AIAA, 2005, 43 (10), 2110-2116. 
[20] FLUENT User Guide Manual 6.2.16, 2004.

[21] Smith B.L. and Glezer A., The Formation and evolution of synthetic jets. Physics of fluids, 1998, 10 (September), pp. 2281-2297.

[22] Yao C.S., Chen F.J. and Neuhart D., Synthetic Jet Flowfeild Database for Computational Fluid Dynamics Validation, AIAA, 2006, 44(12), pp. 3153-3157.

[23] Langley Research Centre Workshop on CFD Validation of Synthetic Jets and Turbulent Separation Control (CFDVAL2004), Williamsburg, Virginia, 2004.

[24] Jagannatha D., Narayanaswamy R., and Chandratilleke T.T, Analysis of a synthetic jet-based electronic cooling module, Numerical Heat Transfer, Part A: Applications, 2009, 56 (3), pp. 211-229.

[25] Chandratilleke T.T, Jagannatha D., and Narayanaswamy R., Heat transfer enhancement in microchannels with cross-flow synthetic jets, International Journal of Thermal sciences, 2010, 49, pp. 504-513. 